\title{
Fault-Tolerant Attitude Control System for a Spacecraft with Control Moment Gyros Using Multi- Objective Optimization
}

\author{
Ai Noumi \\ School of Science for Open and Environmental Systems \\ Keio University \\ Yokohama, Japan \\ Takuya Kanzawa \\ Aerospace Research and Development Directorate \\ Japan Aerospace Exploration Agency \\ Tsukuba, Japan
}

\author{
Misuzu Haruki \\ Aerospace Research and Development Directorate \\ Japan Aerospace Exploration Agency \\ Tsukuba, Japan \\ Masaki Takahashi \\ Department of System Engineering \\ Keio University \\ Yokohama, Japan
}

\begin{abstract}
Recent years have seen a growing requirement for accurate and agile attitude control of spacecraft. To both quickly and accurately control the attitude of a spacecraft, Control Moment Gyros (CMGs) which can generate much higher torque than conventional spacecraft actuators are used as actuators of the spacecraft. The drive on the motors is needed for rapid maneuverability, negatively affecting their life. Thus, in designing spacecraft the conflicting requirements are rapid maneuverability and reduced the drive on motors. Furthermore, the attitude control system needs to be fault-tolerant. The dominant requirement is different for each spacecraft mission, and therefore the relationship between the requirements should be shown. In this study, a design method is proposed for the attitude control system, using multi objective optimization of the skew angle and parameters of the control system. Pareto solutions that can show the relationship between the requirements are obtained by optimizing the parameters. Through numerical analysis, the effect with fault-tolerance and parameter differences for the dominant requirement are confirmed and the method to guide for determining parameters of the attitude control system is established.
\end{abstract}

Keywords-Control Moment Gyros; Spacecraft; Attitude Control; Multi-objective Optimization

\section{INTRODUCTION}

These days spacecraft require rapid rotational maneuverability because of the diversity and complexity of missions. Rapid rotational agility as well as a precision steady attitudinal state are required for the attitude control of spacecraft[1]. Rapid rotational agility as well as a precision steady attitudinal state are required for an attitude control of spacecraft. To meet this demand, Control Moment Gyros (CMGs) are ideal as an attitude control actuator of an agile spacecraft. Compared with previously used actuators, for example Reaction Wheels (RWs), CMGs can effectively generate higher torque. Many methods have been proposed to solve CMG's specific singularity problem[2].
The pyramid-type four-CMG system, as shown in Fig. 1, is commonly used with a skew angle set to 54.74 degree. In actual operation, it is necessary to combine several CMGs for redundancy. Skew angle is usually selected as $\mathbf{5 4 . 7 4}$ degree because the maximum angular momentum for each axis in Fig. 2 is the same. However, it is not necessary for the three axes to have the same angular momentum in the case of a spacecraft such as earth observation satellites whose mission angle is fixed. In fact, the skew angle is set to 30 degree for PleiadesHR1 because a roll slew maneuver is assumed to be the main mission[3].

Therefore the author previously proposed optimizing the skew angle and parameters of the control system in Fig. 3 to achieve the shortest settling time, assuming a specific mission[4]. However, the load on the motor and bearings cannot be ignored for a long term mission because the drive on the gimbal motor is needed to shorten the settling time merely to achieve rapid maneuverability. At the same time reduced drive on $C M G$ is required for spacecraft.

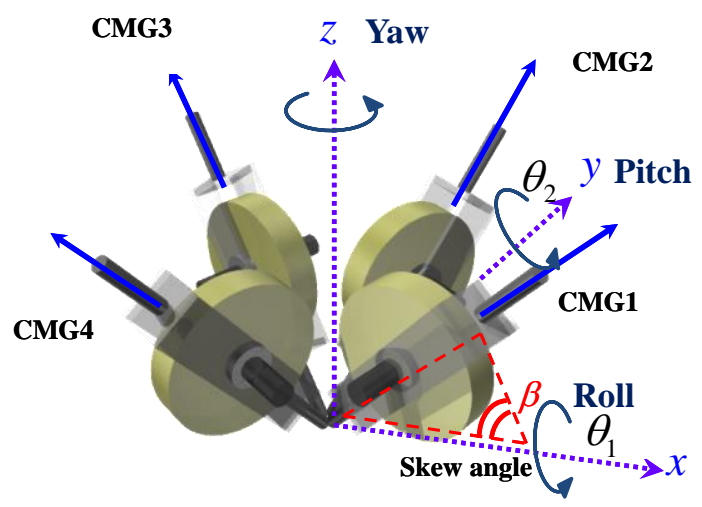

Fig. 1. Skew array CMG system 


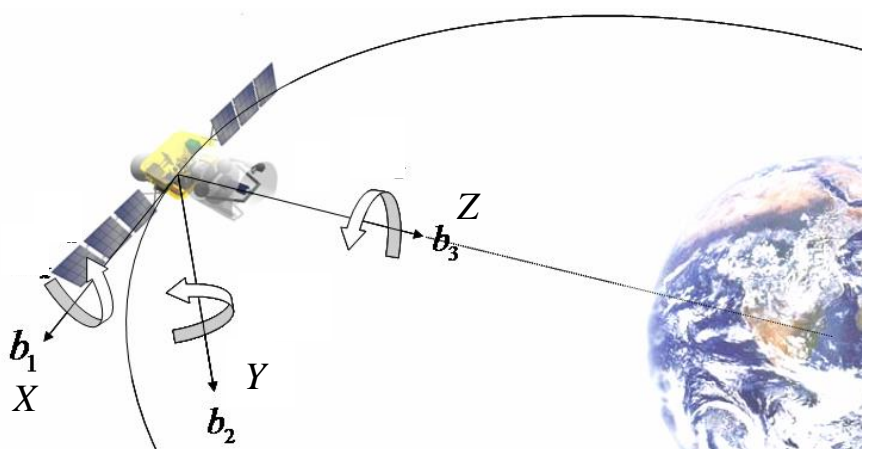

Fig. 2. Coordinate of spacecraft

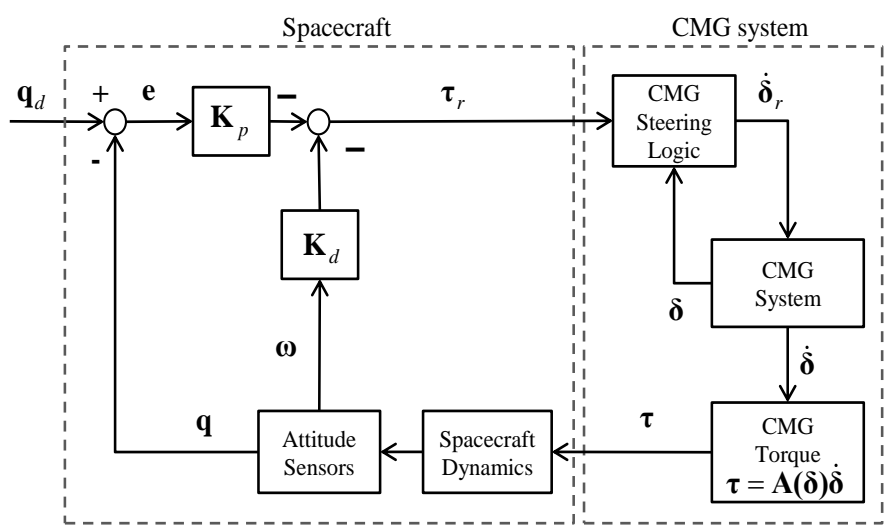

Fig. 3. Attitude control system of agile spacecraft with CMG system

The failure of the ISS CMGs in 2002 was mainly caused by excessive accumulation of the load as a result of metal fatigue of the gimbal axis, especially in the bearing[5],[6]. Therefore, reduced the drive on the gimbal can be effective in extending the operating life of CMGs, as well as reducing power consumption. To achieve rapid maneuverability is important for spacecraft with CMGs but it is not necessary to achieve the shortest settling time because the mission should be achievable within a given preset time. Therefore it is appropriate to design the attitude control system taking into account the conflicting requirements, which are to achieve rapid maneuverability and reduce the drive on the gimbal, for a long operating life.

In this study, multi-objective optimization of skew angle and parameters of control system considering conflicting requirements is proposed. The Pareto solutions considering conflicting objectives are obtained using optimization of parameters. The relationship between requirements and parameters is shown by calculating Pareto solutions from the optimization of parameters. As a specific design method, the settling time and motion of gimbal axes are evaluation criteria when considering rapid maneuverability and reduced the drive on CMG. The combination of parameters that minimizes both evaluation values is obtained by multi objective genetic algorithm (MOGA)[7].

In numerical simulation, the three types of optimization were conducted in addition to the proposed method, as comparative methods. From simulation results, the changes in the parameters with or without consideration of the drive on the gimbal, the effectiveness of optimizing skew angle, effect with fault-tolerance and the parameter differences for the dominant requirement are confirmed. Therefore, the method to guide for determining parameters of the attitude control system is established.

\section{CMG SYSTEM}

\section{A. Schema of CMG}

CMG is an actuator that can generate torque using the gyro effect with a swinging wheel that rotates at a constant rate in a gimbal axis, perpendicular to the axis of wheel rotation. In actual operation, it is necessary to combine several CMGs for redundancy. In this study, an agile spacecraft is considered to have a CMG system that has a pyramid arrangement of four single-gimbal CMGs as shown in Fig. 1.

\section{B. Modeling of CMGs}

A block diagram representation of the CMG-based attitude control system of the agile spacecraft is illustrated in Fig. 3 . When a target angle is required, a torque command vector is calculated using both the current Euler angle and angular velocity vector of the spacecraft $\boldsymbol{\omega}$, which are detected by the spacecraft's own sensors of angular position and velocity. The CMG gimbal angular velocity vector command, which is needed to achieve the torque command, is calculated using the equation of inverse kinematics, named steering logic[8]. Torque is generated from the gimbal angular velocity which, in turn, is generated by activating CMGs to follow the gimbal angular velocity vector command.

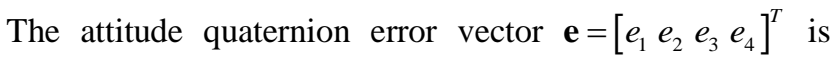
computed using the quaternion of the reference angle of spacecraft $\mathbf{q}_{d}=\left[q_{1 d} q_{2 d} q_{3 d} q_{4 d}\right]^{T}$ and the quaternion of the current angle of the spacecraft $\mathbf{q}=\left[\begin{array}{lll}q_{1} & q_{2} & q_{3} \\ q_{4}\end{array}\right]^{T}$, as follows:

$$
\left[\begin{array}{l}
e_{1} \\
e_{2} \\
e_{3} \\
e_{4}
\end{array}\right]=\left[\begin{array}{cccc}
q_{4 d} & q_{3 d} & -q_{2 d} & -q_{1 d} \\
-q_{3 d} & q_{4 d} & q_{1 d} & -q_{2 d} \\
q_{2 d} & -q_{1 d} & q_{4 d} & -q_{3 d} \\
q_{1 d} & q_{2 d} & q_{3 d} & q_{4 d}
\end{array}\right]\left[\begin{array}{l}
q_{1} \\
q_{2} \\
q_{3} \\
q_{4}
\end{array}\right]
$$

The linear state feedback controller is defined as:

$$
\begin{aligned}
\boldsymbol{\tau}_{r} & =-\mathbf{K}_{p} \mathbf{e}-\mathbf{K}_{d} \boldsymbol{\omega} \\
& =-\left[\begin{array}{cccc}
0 & K_{p} & 0 & 0 \\
0 & 0 & K_{p} & 0 \\
0 & 0 & 0 & K_{p}
\end{array}\right] \mathbf{e}-\left[\begin{array}{ccc}
K_{d} & 0 & 0 \\
0 & K_{d} & 0 \\
0 & 0 & K_{d}
\end{array}\right] \boldsymbol{\omega}
\end{aligned}
$$

where $K_{p}$ is the proportional controller gain, and $K_{d}$ is the derivative controller gain, of the spacecraft and $\boldsymbol{\tau}_{r}$ is the torque command vector.

The angular momentum for four skew type CMGs shown in Fig. 1 is a function depending on the CMG gimbal angle vector $\boldsymbol{\delta}=\left[\begin{array}{llll}\delta_{1} & \delta_{2} & \delta_{3} & \delta_{4}\end{array}\right]^{T}$ as follows: 


$$
\begin{aligned}
\mathbf{h} & =h_{C M G} \sum_{i=1}^{4} \mathbf{H}_{i}\left(\delta_{i}\right) \\
& =h_{C M G}\left[\begin{array}{c}
-c \beta \sin \delta_{1}-\cos \delta_{2}+c \beta \sin \delta_{3}+\cos \delta_{4} \\
\cos \delta_{1}-c \beta \sin \delta_{2}-\cos \delta_{3}+c \beta \sin \delta_{4} \\
s \beta \sin \delta_{1}+s \beta \sin \delta_{2}+s \beta \sin \delta_{3}+s \beta \sin \delta_{4}
\end{array}\right]
\end{aligned}
$$

where $h_{C M G}$ is the angular momentum of the CMG wheel, $\mathbf{H}_{i}(i=1,2,3,4)$ is the angular momentum vector of the $i$ th $\mathrm{CMG}, \beta$ is the skew angle of the four CMGs, $c \beta=\cos \beta$ and $s \beta=\sin \beta$. A time derivative of the $\mathrm{CMG}$ angular momentum vector is given by:

$$
\begin{aligned}
\dot{\mathbf{h}} & =h_{C M G}\left[\begin{array}{cccc}
-c \beta \cos \delta_{1} & \sin \delta_{2} & c \beta \cos \delta_{3} & -\sin \delta_{4} \\
-\sin \delta_{1} & -c \beta \cos \delta_{2} & \sin \delta_{3} & c \beta \cos \delta_{4} \\
s \beta \cos \delta_{1} & s \beta \cos \delta_{2} & s \beta \cos \delta_{3} & s \beta \cos \delta_{4}
\end{array}\right] \dot{\boldsymbol{\delta}} \\
& =\mathbf{A}(\boldsymbol{\delta}) \dot{\boldsymbol{\delta}} \\
& \equiv \boldsymbol{\tau}
\end{aligned}
$$

where $\dot{\boldsymbol{\delta}}=\left[\begin{array}{llll}\dot{\delta}_{1} & \dot{\delta}_{2} & \dot{\delta}_{3} & \dot{\delta}_{4}\end{array}\right]^{T}$ is the CMG gimbal angular velocity vector, $\mathbf{A}$ is a $3 \times 4$ Jacobian matrix, and $\boldsymbol{\tau}$ is the torque vector. From Eq. (4), the gimbal angular velocity vector command is calculated and determines the gimbal angular velocity to generate the torque command. This is called Steering Logic[8] and is an inverse kinematics equation for calculating the gimbal angular velocity vector command. Most simple CMG steering logic uses a pseudo inverse matrix of $\mathbf{A}$ :

$\dot{\boldsymbol{\delta}}_{r}=\mathbf{A}^{T}\left(\mathbf{A} \mathbf{A}^{T}\right)^{-1} \boldsymbol{\tau}_{r}$

where $\dot{\boldsymbol{\delta}}_{r}$ is the gimbal angular velocity command vector, $\mathbf{A}^{+}=\mathbf{A}^{T}\left(\mathbf{A} \mathbf{A}^{T}\right)^{-1}$, which is often referred to as the pseudo inverse steering logic. Most CMG steering laws determine the gimbal rate commands with some variant of the pseudo inverse. If the rank of (A) $<3$ for certain sets of gimbal angles, or, equivalently, the rank of $\left(\mathbf{A} \mathbf{A}^{T}\right)<3$, the pseudo inverse does not exist and the pseudo inverse steering logic encounters singular states. This singular situation occurs when all the individual $\mathrm{CMG}$ torque output vectors are perpendicular to the commanded torque direction. Equally, the singular situation occurs when all the individual CMG momentum vectors have external projections onto the commanded torque vector direction. This is needed to avoid singular states when the chance of failure increases because the value of the gimbal angular velocity vector command is extremely high and would be a strain on the gimbal axis of the CMG at the singularity.

In this study, Generalized Singularity Robust (GSR)Inverse logic[8],[9], proposed by Bong Wie, is used for the steering logic. This is a method for avoiding a singularity by generating a torque with given gimbal angular velocity command, even in a singular situation, as follows. The GSRInverse steering logic can be represented as:

$\dot{\boldsymbol{\delta}}_{r}=\mathbf{A}^{\#} \boldsymbol{\tau}_{r}$

$$
\begin{aligned}
\mathbf{A}^{\#} & =\mathbf{A}^{T}\left[\mathbf{A} \mathbf{P} \mathbf{A}^{T}+\lambda \mathbf{I}_{4}\right]^{-1} \mathbf{A}^{T} \mathbf{P} \\
& =\mathbf{A}^{T}\left[\mathbf{A} \mathbf{A}^{T}+\lambda \mathbf{P}^{-1}\right]^{-1} \\
& =\mathbf{A}^{T}\left[\mathbf{A} \mathbf{A}^{T}+\lambda \mathbf{E}\right]^{-1} \\
\mathbf{P}^{-1} & \equiv \mathbf{E}=\left[\begin{array}{ccc}
1 & \varepsilon_{3} & \varepsilon_{2} \\
\varepsilon_{3} & 1 & \varepsilon_{1} \\
\varepsilon_{2} & \varepsilon_{1} & 1
\end{array}\right]>\mathbf{0}
\end{aligned}
$$

where $\varepsilon_{i}=\varepsilon_{0} \sin \left(\omega t+\phi_{i}\right)(i=1,2,3), \lambda=\lambda_{0} \times \mathrm{e}^{-\mu \mathrm{m}^{2}}, \omega=\pi / 2$, $\phi_{i}=(1-i) \times(\pi / 2)(i=1,2,3)$.

$t$ is the current time, and $\mathrm{m}=\sqrt{\operatorname{det}\left(\mathbf{A A}^{T}\right)}$ is the singularity measure. $\varepsilon_{0}, \lambda_{0}$ and $\mu$ are constant parameters to be properly selected. The GSR-Inverse logic is used for singularityavoidance steering logic in this study.

The equation to calculate the gimbal angular acceleration of $i$ th CMG $\ddot{\delta}_{i}$ is obtained from:

$$
\ddot{\delta}_{i}=K_{g}\left(\dot{\delta}_{r i}-\dot{\delta}_{i}\right) / J \quad(i=1, \cdots, 4)
$$

where $K_{g}$ is the feedback gain of the CMG control system, $\dot{\delta}_{i}, \dot{\delta}_{r i}$ are the gimbal angular velocity and command of $i$ th CMG and $J$ is the inertia moment matrix of the CMG wheel.

\section{DESIGN OF A FALUt TOLERANT ATtitude CONTROL SYSTEM CONSIDERING CONFILICTING REQUIREMENTS}

The purpose of this chapter is to describe a specific design method for a fault-tolerant attitude control system, considering conflicting requirements.

Initially, the parameters to be optimized are shown. Second, the conflicting requirements, such as rapid maneuverability and reduced drive on gimbals, are defined as the evaluation functions. Finally, the optimization of the parameters is defined using the Multi Objective Genetic Algorithm (MOGA) taking fault-tolerance into consideration.

\section{A. Design Parameters}

\section{1) Skew angle}

The maximum angular momentum of each axis can be changed by changing skew angle $\beta$ in Fig. 1. For a typical pyramid configuration of four single-gimbal CMGs with a skew angle of $\beta$, the angular momentum for the three axes $\mathbf{h}=\left[\begin{array}{lll}h_{x} & h_{y} & h_{z}\end{array}\right]^{T}$ can be obtained analytically as[3],[9]:

$$
\begin{aligned}
& h_{x}=\frac{c \beta\left(-s \beta u_{z}+c \beta u_{x}\right)}{n_{1}}+\frac{u_{x}}{n_{2}}+\frac{c \beta\left(s \beta u_{z}+c \beta u_{x}\right)}{n_{3}}+\frac{u_{x}}{n_{4}} \\
& h_{y}=\frac{u_{y}}{n_{1}}-\frac{c \beta\left(s \beta u_{z}-c \beta u_{y}\right)}{n_{2}}+\frac{u_{y}}{n_{3}}+\frac{c \beta\left(s \beta u_{z}+c \beta u_{y}\right)}{n_{4}}
\end{aligned}
$$




$$
\begin{aligned}
& h_{z}=\frac{s \beta\left(-c \beta u_{x}+s \beta u_{z}\right)}{n_{1}}+\frac{s \beta\left(s \beta u_{z}-c \beta u_{y}\right)}{n_{2}}+ \\
& \frac{s \beta\left(s \beta u_{z}+c \beta u_{x}\right)}{n_{3}}+\frac{s \beta\left(s \beta u_{z}+c \beta u_{y}\right)}{n_{4}} \\
& n_{1}= \pm \sqrt{1-\left(s \beta u_{x}+c \beta u_{z}\right)^{2}} \\
& n_{2}= \pm \sqrt{1-\left(s \beta u_{y}+c \beta u_{z}\right)^{2}} \\
& n_{3}= \pm \sqrt{1-\left(-s \beta u_{x}+c \beta u_{z}\right)^{2}} \\
& n_{4}= \pm \sqrt{1-\left(s \beta u_{y}+c \beta u_{z}\right)^{2}}
\end{aligned}
$$

where $u_{x}=\sin \theta_{2}, u_{y}=-\sin \theta_{1} \cos \theta_{2}$ and $u_{z}=\cos \theta_{1} \cos \theta_{2}$. $\theta_{1}$ and $\theta_{2}$ are the rotation angles of two successive rotations about the $x$ and $y$ axes. From these equations, it is apparent that the maximum angular momentum is directly related to the skew angle $\beta$.

Fig. 4 shows the relationship between the skew angle and the maximum angular momentum $\mathbf{H}=\left[H_{x} H_{y} H_{z}\right]^{T}$ for each axis in normal time when the skew angle was changed from 0 degree to 90 degree every 10 degree and 54.74 degree, which is commonly used. From Fig. 4, it can be seen that the maximum angular momentums of the roll and pitch axes decrease, whereas the maximum angular momentum of the yaw axis increases with the increasing skew angle. Furthermore, it is also apparent that the maximum angular momentums of the three axes are almost the same when the skew angle is 54.74 degree. However, the skew angle needs to be designed taking into consideration the requirements for this study, which are rapid maneuverability and reduced drive on the gimbals. Moreover, the conventional design only considers normal situations, whereas the control system should be designed to consider fault-tolerance when failure of CMGs has been reported during the operation.

A method for dynamically changing the skew angle when the spacecraft is in use has also been proposed[10],[11] because the maximum angular momentum of each axis can be changed by changing the skew angle, which is a valid method when using CMGs. However, the design of a unique skew angle before the launch, is proposed in this study, because potential failure of the added moving element must be taken into consideration when dealing with the failure of a CMG. In this study, it is assumed that one CMG can be shut down entirely in use.

It is apparent that $\mathrm{CMG}$ shutdown can be classified into two patterns, the failure of $\mathrm{CMG} 1$ or $\mathrm{CMG} 3$, or failure of CMG 2 or CMG 4. Figs. 5 and 6 show the relationships between the skew angle and the maximum angular momentum in each situation. These figures show that the maximum angular momentums for every axis decrease than when the CMGs are functioning normally. Moreover, it is the same as the normal situation in that the maximum angular momentums of the roll and pitch axes decrease, while the maximum angular momentum of the yaw axis increases with an increasing skew angle. Comparing the failure of $\mathrm{CMG} 1$ or $\mathrm{CMG} 3$ with the failure of CMG 2 or $\mathrm{CMG} 4$, it is apparent that the values of $H_{x}$ and $H_{y}$ switch place, although the value of $H_{z}$ remains the same, which is verified by a deformation Eqs (10) to (16). For that reason, three situations, normal, failure of CMG 1 and failure of CMG 2, are dealt with in this study.

\section{2) Parameters of the control system}

In this study, both the skew angle and parameters of control system are tuned simultaneously because there is the possibility that the parameters of system, which can achieve the preset goal within target time, can be changed depending on the skew angle.

The parameters to be designed are the gains of the spacecraft attitude control system $K_{p}$ and $K_{d}$, the gain of the CMG control system $K_{g}$, the parameters of the GSR-Inverse logic, $\lambda_{0}, \varepsilon_{0}$ and $\mu$.

\section{B. Method for design of a fault-tolerant attitude control system considering the conflicting requirements}

In this section, the method to determine skew angle and parameters of the control system using MOGA is outlined. As shown in the previous section, the unique skew angle and the six parameters of the control system which are appropriate to each situation are optimized.

First, several initial chromosomes are generated for 19 parameters, as shown in Fig. 7. They contain the gains of the spacecraft attitude control system $K_{p}$ and $K_{d}$, the gain of the CMG control system $K_{g}$, the parameters of the GSR-Inverse $\operatorname{logic} \lambda_{0}, \varepsilon_{0}$, and $\mu$ in each of the three situations and the unique skew angle.

$$
\begin{aligned}
& 10 \leq \beta \leq 55 \\
& 30 \leq K_{p} \leq 200 \\
& 50 \leq K_{d} \leq 250 \\
& 0.27 \leq K_{g} \leq 0.285 \\
& -4 \leq \log _{10} \lambda_{0} \leq 4 \\
& -4 \leq \log _{10} \varepsilon_{0} \leq 4 \\
& -4 \leq \log _{10} \mu \leq 4
\end{aligned}
$$

The range of the skew angle is set from 10 degree to 55 degree which provides the large maximum angular momentum for the roll and pitch axes from the results in Fig. 4. 


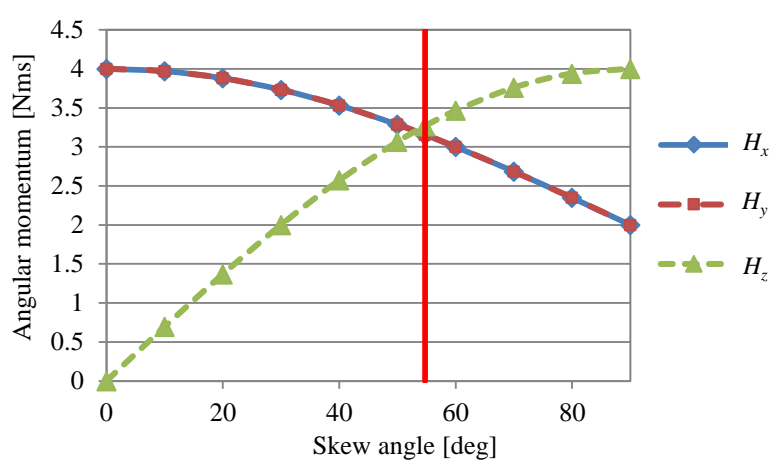

Fig. 4. Relationship between the skew angle and the maximum angular momentum

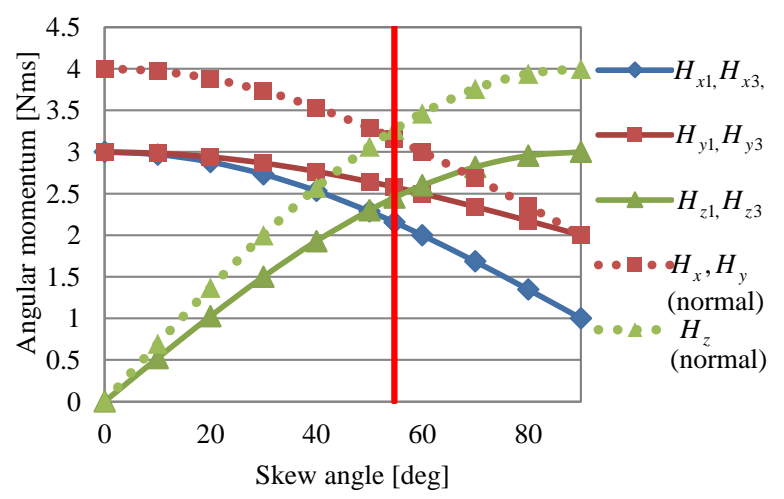

Fig. 5. Relationship between the skew angle and the maximum angular momentum (Failure of CMG 1 or CMG 3)

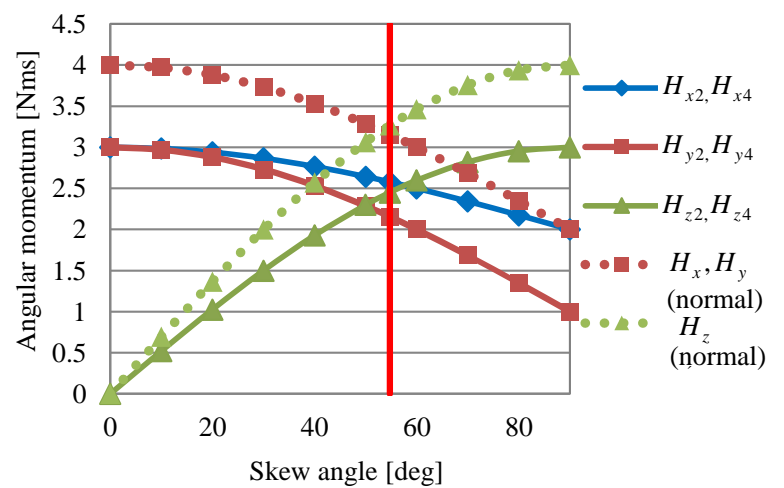

Fig. 6. Relationship between the skew angle and the maximum angular momentum (Failure of CMG 2 or CMG 4)

The settling time $t_{j}$ and drive on the gimbal (gimbal angle, velocity and acceleration) in each of the three situations are evaluated for each chromosome. Where $j$ means the situation, $j=1$ is the normal situation, $j=2$ is the situation of a failure of CMG 1 and $j=3$ is the situation of a failure of CMG 2 . The settling time and drive on the gimbal for each chromosome are defined as $f_{1}$ and $f_{2}$, and these are the summation of the evaluation value for each situation. Here the weight of the evaluation value for each situation $a_{j}$ is designed according to the preferred situation.In this paper, $a_{j}=1$ to deal every situation equivalently.

$$
f_{1}=\sum_{j=1}^{3} f_{1 j} / \sum_{j=1}^{3} a_{j}
$$$$
f_{1 j}=a_{j}\left(t_{j} / t_{\max }\right)
$$

$$
f_{2}=\sum_{j=1}^{3} f_{2 j} / \sum_{j=1}^{3} a_{j}
$$

$$
f_{2 j}=a_{j}\left(f_{\delta}+f_{\dot{\delta}}+f_{\ddot{\delta}}\right)
$$

$$
f_{\delta}=\frac{1}{4 t_{j}} \sum_{i=1}^{4} \sum_{k=0}^{t_{j} / d t}\left(\frac{\delta_{i}(k d t)}{\delta_{\mathrm{m}}}\right)^{2}
$$

$$
f_{\dot{\delta}}=\frac{1}{4 t_{j}} \sum_{i=1}^{4} \sum_{k=0}^{t_{j} / d t}\left(\frac{\dot{\delta}_{i}(k d t)}{\dot{\delta}_{\mathrm{m}}}\right)^{2}
$$

$$
f_{\ddot{\delta}}=\frac{1}{4 t_{j}} \sum_{i=1}^{4} \sum_{k=0}^{t_{j} / d t}\left(\frac{\ddot{\delta}_{i}(k d t)}{\ddot{\delta}_{\mathrm{m}}}\right)^{2}
$$

where $\delta_{i}, \dot{\delta}_{i}$ and $\ddot{\delta}_{i}$ are the $i$ th gimbal angle, velocity and acceleration. $\delta_{\text {max }}=6, \dot{\delta}_{\text {max }}=1$ and $\ddot{\delta}_{\text {max }}=3$ because the values of the terms should be the same. In Eqs (21) to (23), the

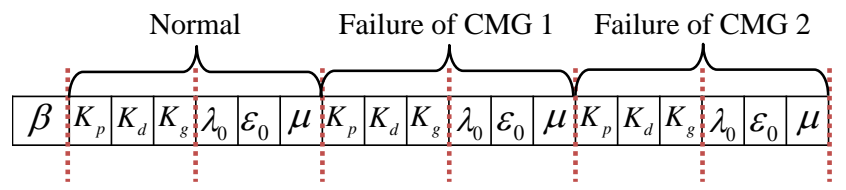

Fig. 7. Gene of an individual for genetic algorithm

summation of the data for each gimbal $(i=1, \cdots, 4)$ is divided by $4 t$, which means the evaluation value is the average of the drive on the gimbal of a CMG for $1 \mathrm{~s}$.

Pareto solutions are obtained by optimizing the combination of parameters which minimizes both evaluation values with the evaluation function $f_{1}$ for the settling time and the evaluation function $f_{2}$ for the drive on the gimbal using MOGA[7].

In this study, the mission assumed is a rest-to-rest maneuver, which means that the spacecraft body must rest at the beginning and end of a maneuver to observe a ground target, and is analyzed assuming a rigid body spacecraft. The spacecraft model is assumed to be a middle-sized earthobserving satellite whose directional axis is the yaw axis. The parameters used in the numerical simulations are given in TABLE II. The mission assumed for the analysis is to perform a 60 degree roll (cross-track) slew maneuver based on an actual earth-observing satellite, the ALOS-2[12]. The settling time is 
defined as when the Euler angle has settled to the target maneuver angle within \pm 0.01 degree, and is the same for the pitch and yaw axes. The parameters of the CMG are the same as the CMG, 15-4S, in Pleiades-HR1 manufactured by Astrium[13].

\section{NUMERICAL ANALYSIS}

Fig. 8 shows the Pareto solutions obtained using MOGA, referred to in the last section.

The three types of optimization were conducted in addition to the proposed method, as comparative methods. TABLE III. shows the comparison of each method. Fig. 8 shows the comparative results from the three methods.

\section{A. The changes in the parameters with or without consideration of the drive on the gimbal}

In this section, the change in the optimized parameters when the evaluation function of the drive on the gimbal is or is not considered is compared for Methods 1 and 3. TABLE IV. shows the average and standard deviation of parameters for normal situation in each method.

From Fig. 8, the solution which satisfies smaller drive on gimbal than Method 1 when settling time is same as Method 1 is obtained in Method 3. From TABLE IV. smaller skew angle than for Method 3 is obtained in Method 1 to make settling time shorter because the angular momentum is larger for roll axis. On the other hand, the solution which satisfies smaller drive on the gimbal with same settling time than Method 1 is obtained even though skew angle in Method 3 is larger than in Method 1. It is because that appropriate parameters of the control system which satisfies both requirements are obtained using the evaluation function for the drive on the gimbal in addition to for the settling time.

\section{B. Difference with and without optimizing skew angle}

In this section, comparing Methods 2 and 3, the changes in the values of the evaluation functions are discussed when the skew angle is optimized and when it is set at 54.74degree.

In Method 3, skew angle is selected at $31.98 \pm 7.64$ degree which is smaller than 54.74 degree. It is noted that the settling time could have been shorter by optimizing the skew angle compared with when it was set at 54.74 degree. This is because a larger torque can be generated when skew angle is smaller as shown in the previous section. From this result, making the skew angle smaller can make the settling time shorter, but the drive on the gimbal could be greater when the settling time is longer for a skew angle of 54.74 degree. It is confirmed that skew angle can be designed according to requirements.

\section{Effect with or without consideration of fault tolerance}

In this section, comparing Methods 2 and 3, the effect with or without consideration of fault-tolerance is discussed.

From TABLE IV. skew angle is smaller when considering fault-tolerance. It can be assumed that smaller skew angle is obtained with fault-tolerance because the maximum angular momentum is smaller in failure situations than in a normal situation. Moreover, standard deviations of parameters in Method 4 are smaller than in Method 3. Fig. 9 shows the
Pareto solutions for normal situation in Methods 3 and 4. From Fig. 9, the range of solution in Method 4 is smaller than in Method 3. It is assumed that range of solution for normal situation in Method 4 is smaller because of the consideration of fault-tolerance. Therefore, wider design of the attitude control system in normal situation is available without consideration of fault-tolerance.

\section{Parameter differences for the dominant requirement}

In this section, parameter differences in Method 4 for the dominant requirement are discussed. Fig. 10 shows Pareto solution obtained by Method 4. The parameters in the normal situation for the three solutions in Fig. 10 which correspond to the dominant requirement as a discriminative solution of Pareto solutions in Method 4 are discussed:

1) Solution in which rapid maneuverability is dominant,

2) Solution in which both requirements are equivalently dominant,

3) Solution in which reduced the drive on the gimbal is dominant

TABLE V. shows the values of parameters in each solution. Figs. 11 to 13 show the time histories of the singularity parameter, gimbal velocity and torque as three solutions It is assumed that the solution satisfying the requirement of rapid maneuverability because the gimbal is driven rapidly in a singularity situation as a result of the combination of parameters of GSR Inverse logic $\lambda_{0}, \varepsilon_{0}, \mu$, is obtained in I. Therefore torque errors for pitch and yaw axes are larger than other solutions to avoid singularity as quick as possible. In addition, it is assumed that the solution satisfying the requirement of reduced drive on the gimbal because the gimbal is driven slowly even in a singularity situation as a result of the combination of parameters of GSR Inverse logic $\lambda_{0}, \varepsilon_{0}, \mu$, is obtained in III. From these results, it is verified that the faulttolerant attitude control system which satisfies the dominant requirement by changing characteristic of the control system by parameters of the control system.

TABLE I. THE VALUES OF THE SyMBols USED IN THE EVALUATION FUNCTIONS

\begin{tabular}{|l|l|}
\hline \multicolumn{1}{|c|}{ Symbols } & \multicolumn{1}{c|}{ Values } \\
\hline$t_{\max }$ & 40 \\
\hline$\delta_{\mathrm{m}}$ & 6 \\
\hline$\dot{\delta}_{\mathrm{m}}$ & 1 \\
\hline$\ddot{\delta}_{\mathrm{m}}$ & 3 \\
\hline
\end{tabular}

TABLE II. PARAMETERS AND VALUES FOR THE NUMERICAL SIMULATIONS

\begin{tabular}{|l|c|l|}
\hline \multicolumn{1}{|c|}{ Parameters } & Symbols & \multicolumn{1}{c|}{ Values } \\
\hline Inertia moment of spacecraft & $\mathbf{I}_{s}$ & $\operatorname{diag}(5000,5000,3000) \mathrm{kgm}^{2}$ \\
\hline $\begin{array}{l}\text { Inertia moment of CMG } \\
\text { wheel }\end{array}$ & $J$ & $0.19 \mathrm{kgm}^{2}$ \\
\hline Angular momentum of CMG & $h_{C M G}$ & $75 \mathrm{Nms}$ \\
\hline Max. gimbal rate & $\dot{\delta}_{\max }$ & $1.0 \mathrm{rad} / \mathrm{s}$ \\
\hline Max. gimbal acceleration & $\ddot{\delta}_{\max }$ & $3.0 \mathrm{rad} / \mathrm{s}^{2}$ \\
\hline Control cycle & $d t$ & $0.01 \mathrm{~s}$ \\
\hline
\end{tabular}


TABLE III. COMPARISON OF EACH METHOD

\begin{tabular}{|l|c|c|c|c|}
\hline \multirow{2}{*}{ Method } & \multicolumn{2}{|c|}{ Evaluation function } & \multirow{2}{*}{$\begin{array}{c}\text { Fault- } \\
\text { tolerance }\end{array}$} & $\begin{array}{c}\text { Optimizing } \\
\text { skew angle }\end{array}$ \\
\cline { 2 - 5 } & $\begin{array}{c}\text { Settling } \\
\text { time }\end{array}$ & $\begin{array}{c}\text { Drive on } \\
\text { gimbal }\end{array}$ & $\times$ & $\circ$ \\
\hline 1 & $\circ$ & $\times$ & $\times$ & 54.74 degree \\
\hline 2 & $\circ$ & 0 & & $\circ$ \\
\hline 4 (Proposed) & $\circ$ & 0 & $\times$ & 0 \\
\hline
\end{tabular}

TABLE IV. AVERAGE AND STANDARD DEVIATION OF PARAMETERS FOR NORMAL SITUATION IN EACH METHOD

\begin{tabular}{|l|c|c|c|c|}
\hline \multirow{2}{*}{$\begin{array}{c}\text { Paramete } \\
\mathbf{r}\end{array}$} & \multicolumn{4}{|c|}{ Average \pm Standard deviation } \\
\cline { 2 - 5 } $\begin{array}{c}\text { Skew } \\
\text { angle } \\
{[\mathrm{deg}]}\end{array}$ & 18.1 & 54.74 & $31.98 \pm 7.64$ & $28.77 \pm 0.58$ \\
\hline$K_{p}$ & 171.15 & $101.97 \pm 28.38$ & $70.33 \pm 15.53$ & $92.37 \pm 3.77$ \\
\hline$K_{d}$ & 299.1 & $282.68 \pm 15.59$ & $211.27 \pm 28.38$ & $247.10 \pm 4.24$ \\
\hline$K_{g}$ & 0.284 & $0.281 \pm 0.004$ & $0.277 \pm 0.0022$ & $0.280 \pm 0.003$ \\
\hline $\log _{10} \lambda_{0}$ & -1.84 & $-0.21 \pm 0.57$ & $-0.83 \pm 0.25$ & $-0.92 \pm 0.11$ \\
\hline $\log _{10} \varepsilon_{0}$ & 0.19 & $-0.99 \pm 0.25$ & $-1.22 \pm 0.34$ & $-1.12 \pm 0.15$ \\
\hline $\log _{10} \mu$ & -1.42 & $-1.66 \pm 0.72$ & $-0.11 \pm 0.91$ & $0.97 \pm 0.32$ \\
\hline
\end{tabular}

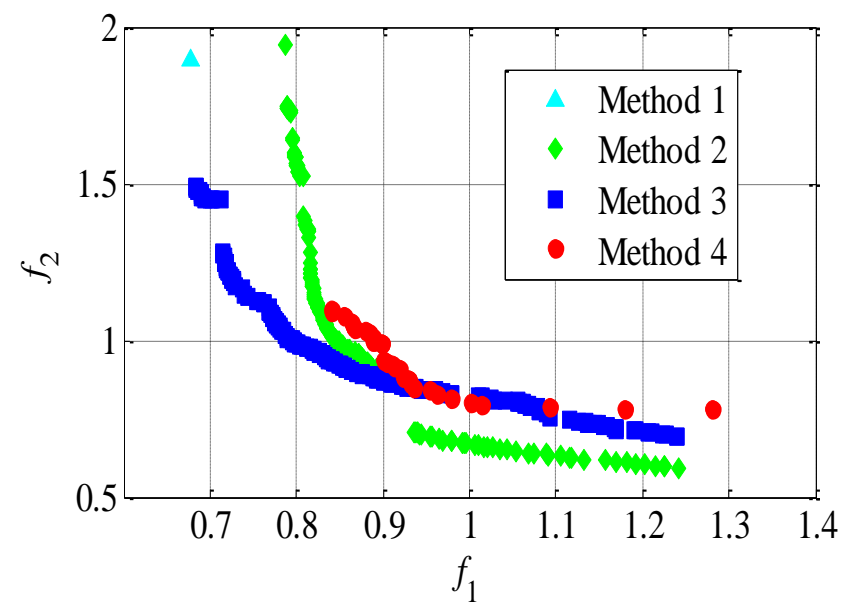

Fig. 8. Pareto solutions

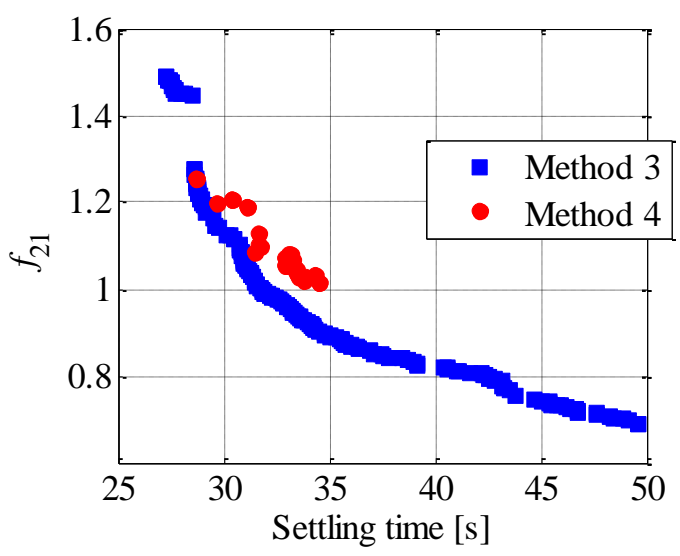

Fig. 9. Pareto solutions (Normal situation in Methods 3 and 4)

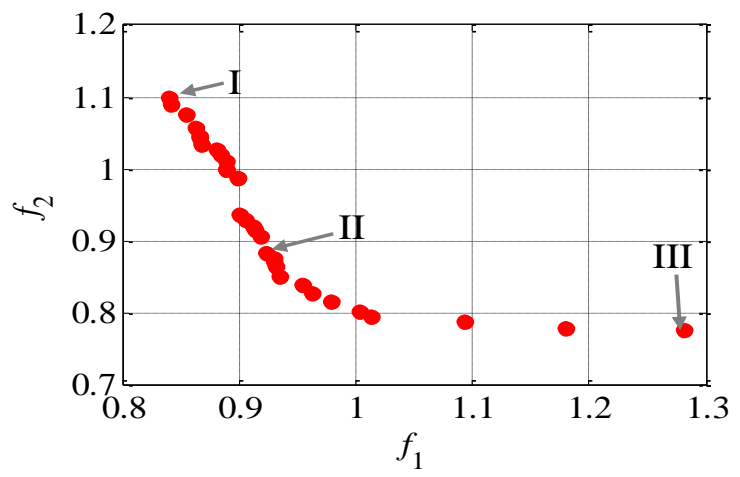

Fig. 10. Pareto solutions (Method 4)

\section{CONCLUSIONS}

This study proposed multi-objective optimization of the skew angle and parameters of the control system to design of a fault-tolerant attitude control system that would take into account conflicting requirements for a spacecraft with a skew array of Control Moment Gyros. The relationship between the requirements and the relationship between the requirements and parameters can be shown by calculating the Pareto solutions which is a class of solutions that comprehensively consider conflicting requirements.

From simulation results, the changes in the parameters with or without consideration of the drive on the gimbal, the effectiveness of optimizing skew angle, effect with faulttolerance and the parameter differences for the dominant requirement were confirmed. Therefore, the method to guide for determining parameters of the attitude control system was established. To optimize the parameters considering constraint of spacecraft and to verify the effectiveness of the proposed method using an actual operation are the future works. 
TABLE V. The VAlues of Parameters in Each Solution (Method 4)

\begin{tabular}{|l|c|c|c|c|c|c|c|c|c|}
\hline & $\begin{array}{c}\text { Settling } \\
\text { time [s] }\end{array}$ & $f_{2}$ & $\begin{array}{c}\text { Skew angle } \\
{[\mathbf{d e g}]}\end{array}$ & $K_{p}$ & $K_{d}$ & $K_{g}$ & $\log _{10} \lambda_{0}$ & $\log _{10} \varepsilon_{0}$ & $\log _{10} \mu$ \\
\hline I & 28.71 & 1.26 & 28.06 & 89.93 & 247.07 & 0.280 & -0.86 & -0.95 & 0.83 \\
\hline II & 32.96 & 1.05 & 28.40 & 90.63 & 245.81 & 0.277 & -0.97 & -1.15 & 0.87 \\
\hline III & 34.32 & 1.03 & 29.31 & 92.50 & 252.87 & 0.280 & -1.09 & -1.10 & 0.76 \\
\hline
\end{tabular}

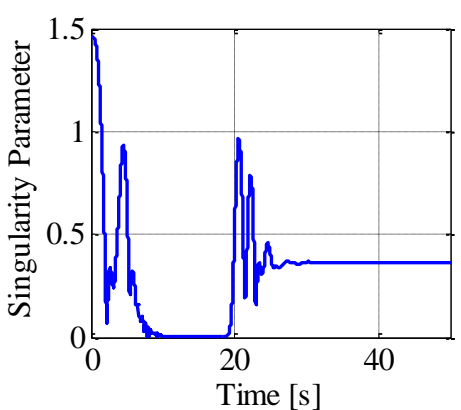

(a) I

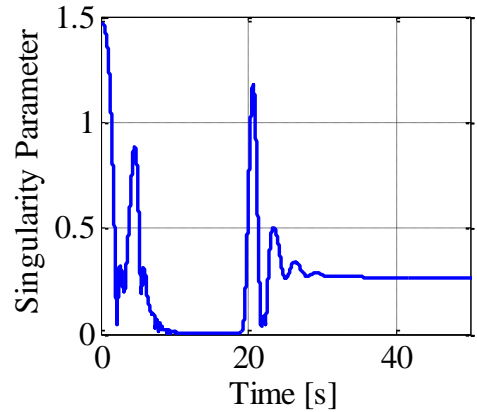

(b) II

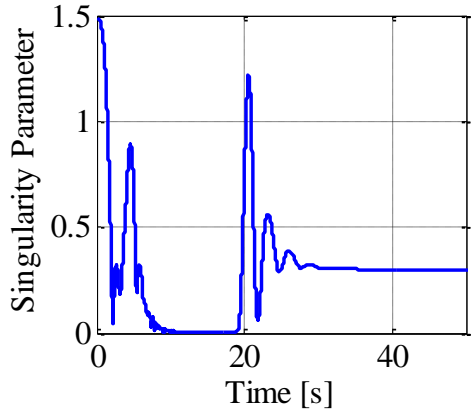

(c) III

Fig. 11. Time histories of the singularity parameters

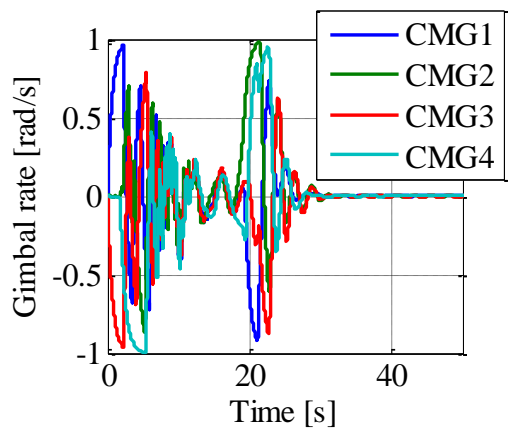

(a) I

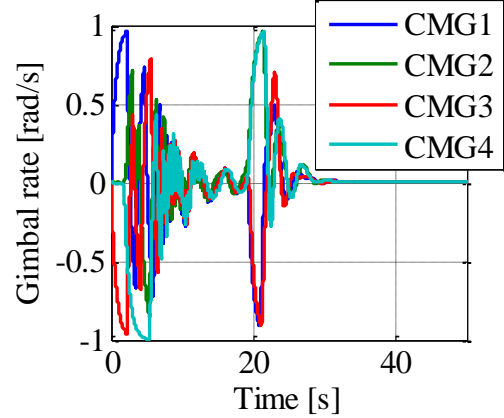

(b) II

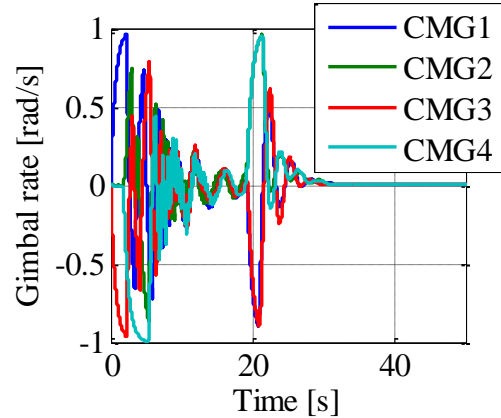

(c) III

Fig. 12. Time histories of gimbal angle velocity

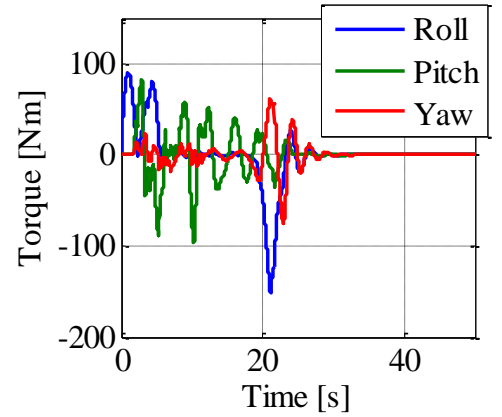

(a) I

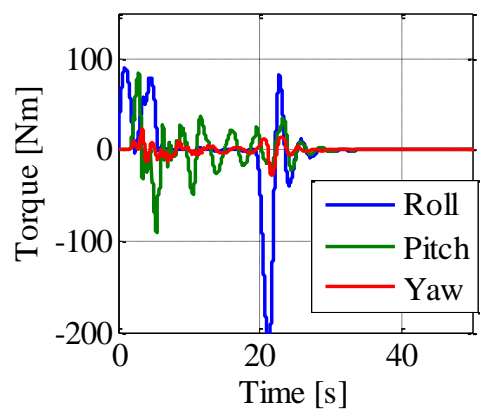

(b) II

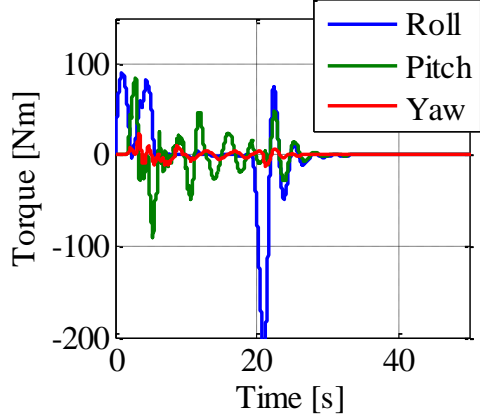

(c) III

Fig. 13. Time histories of torque

REFERENCES

[1] The Mechanical Social Systems Foundation, "Research on the realization of Earth observation satellite monitoring with advanced maneuver capability", 2009, pp. $57-61$.

[2] Kurokawa, H., "A geometric study of single gimbal control moment gyros - singularity problems and steering law -", Report of Mechanical Engineering Series, No.175, 1998.

[3] Wie, B., Space Vehicle Dynamics and Control Second Edition, AIAA Education Series, American Institute of Aeronautics and Astronautics, Inc., 2008.
[4] Noumi, A., Takahashi, M., "Fault-tolerant attitude control systems for a satellite equipped with control moment gyros", AIAA Guidance, Navigation, and Control Conference, AIAA, Boston, 2013, AIAA2013 5119.

[5] Burt, R. R., Loffi, R. W., "Failure analysis of international space station control moment gyro", Proceedings of the $10^{\text {th }}$ European Space Mechanisms and Tribology Symposium, Spain, 2003, pp. 13 - 25.

[6] Gurrisi, C., Seidel, R., Dickerson, S., Didziulis, S. and Frantz, P., Ferguson, K., "Space station control moment gyroscope lessons 
learned", Proceedings of the 40th Aerospace Mechanisms Symposium, 2010, pp. 161 - 176

[7] Deb, K., Pratap, A., Agarwal, S. and Metarivan, T., "A fast and elitist multiobjective genetic algorithm: NSGA- II", IEEE Transaction on Evolutionary Computation, Vol.24, No.5, 2001, pp. 865- 872.

[8] Wie, B., Bailey, D. and Heiberg, C., "Singularity robust steering logic for redundant single-gimbal control moment gyros", Journal of Guidance, Control, Dynamics, Vol.24, No.5, 2001, pp. 865 - 872.

[9] Wie, B., "Singularity escape/avoidance steering logic for redundant single-gimbal control moment gyros", Journal of Guidance, Control, Dynamics, Vol. 28, No.5, 2005, pp. 948 - 956.

[10] Kojima, H., Matsuda, N., and Takada, K., "Adaptive skewing pyramidtype CMGs for fast attitude maneuver", Transactions of Japan Society for Aeronautical and Space Sciences, Space Technology, Japan, Vol.7, 2009, pp. 19 - 24.

[11] Kojima, H., "Singularity analysis and steering control laws for adaptiveskew pyramid-type control moment gyros", Acta Astronautica, Vol.85, 2013, pp. 120 - 137.

[12] Kanzawa, T., Iwata, T., Arikawa, Y., Natori, T., "Attitude and orbit control system of the advanced land obserbing satellite-2 (ALOS-2)", Proceeding of 55th Space Science and Technology Conference, The Japan Society for Aeronautical and Space Sciences, Ehime, 2011, JSASS-2011-4242.

[13] Defendini, A., Faucheux, P., Guay, P., Morand, J., and Heimel, H. "A compact CMG products for agile satellites", Proceedings of the $10^{\text {th }}$ European Space Mechanisms and Tribology Symposium, Spain, 2003, pp. $27-31$ 